\title{
AN EXPERIMENTAL ENVIRONMENT FOR DISTRIBUTED INTELLIGENT NETWORK SERVICES
}

\author{
Bilhanan Silverajan, Jarmo Harju \\ Dept. of Information Technology, Tampere University of Technology, P.O. Box 553, FIN-33101 \\ Tampere, Finland \\ \{bilhanan|harju\}@cs.tut.fi
}

Keywords: IN Service Creation Frameworks, Distributed Component-based Systems, Design Patterns, CORBA

Abstract: This paper discusses an experimental lightweight object oriented environment aimed towards the rapid prototyping, testing and deploying of distributed IN services using CORBA. Well-known design patterns have been adapted to implement as well as interwork with CORBA enabled components. Synchronous calls are used for CORBA object requests, while reliable asynchronous delivery such as that needed for all protocol specific transmission is provided via a minimal core subset of an event-based framework known as OVOPS. Thus, no additional CORBA service for messaging or event-handling is needed. The described environment will ensure the consistent creation of IN services as well as needed protocols through reusable object-oriented components communicating over a heterogeneous network. The IN services created would become independent of the underlying protocol which is used to communicate with the other service entities across the network, providing the advantage of being able to run the service over multiple protocols by isolating network specific information flow from service specific information flow.

\section{INTRODUCTION}

Service Creation, when used with telecommunications technically comprises the processes of harnessing technological methods to facilitate the rapid prototyping, developing, testing, deploying and subsequent 
maintaining of services. The field of Intelligent Networks places extreme demands on systems in terms of design, flexibility, scalability and performance, while inherently remaining a highly distributed model. It is thus only logical that such systems evolve to become heavily influenced by advances in distributed processing, especially CORBA.

Also one cannot build software-based telecommunications switching or control products without taking into account the quality and reliability of services the underlying protocols provide. The current trend in IN services is clearly evolving towards decoupling Service Creation and Service Execution environments from the underlying switch networks. A GSM Phase 2+ standard called CAMEL already defines the IN-GSM service interoperation, which allows mobile subscribers to access IN services [1]. Within the area of Intelligent Network Service Creation, there is always an implicit need to constantly reconcile many areas of service and protocol engineering. Although the service designer should not concern himself with signalspecific issues, protocols may change or evolve. Any framework used for service creation should thus be able to address this need to work at the protocol specific aspects as well, so that the same IN services can utilize several types of varying underlying protocol services.

However, at times standards-compliant implementations resulting from applying the multi-plane Intelligent Network specifications are cumbersome or not even possible, leading to many vendor-specific methods of service creation. This in turn directly warrants a need to design a simple, consistent and easy-to-use open framework or environment which, while still supporting rapid service development, can be used in a distributed environment in a standards-conformant manner to reap the advantage of load-processing over multiple machines.

Such an environment was successfully designed by building an experimental IN-specific framework which uses a few core components of an asynchronous event-based protocol implementation environment, OVOPS as a foundational basis. Enough parallels were seen in the areas of protocol implementation and IN Service Creation to be able to apply software practices and techniques learnt in the former to build a framework for the latter.

OVOPS though, only provides simple distribution chiefly through socket based communication techniques without any CORBA-based support for distributed computing. However, the OVOPS I/O Handler's principle of operation closely corresponds to the Reactor Pattern [2] as presented by Schmidt. This allowed the I/O handling of OVOPS to be extended to support CORBA invocations by introducing OVOPS specific device classes with functionalities appropriate to different ORBS. These can then handle 
CORBA events external to the OVOPS system but trapped by the ORBs, as described in [3].

Thus the resulting framework is able to provide reliable native support for both the asynchronous and synchronous models of communication, the former for protocol specific transmission, the latter for making object calls for service execution while remaining CORBA compliant. This implies a reduced overhead for CORBA services such as the Event Service [4] and other services such as the Messaging and Notification Services [5], [6] that are being actively studied within the OMG.

\section{THE FRAMEWORK ARCHITECTURE}

The diagram in Figure 1 illustrates the framework which serves as an environment for both service creation and service execution. A Service Control platform provides a Service Logic Execution Environment (SLEE) within which Service Logic Processing Programs (SLPs) run to provide pertinent service processing. An SLP is a service application program invoked by the SLEE and is used to realize service processing under the control of the SLEE. The simultaneous invocation and execution of multiple SLPs are also managed by the SLEE. When an SLP is selected and invoked, it is referred to as a Service Logic Processing Program Instance (SLPI). In contrast to an SLP, a corresponding SLPI is a dynamic entity that actively controls the flow of service execution. This flow of service execution is broken down into a sequence of Service Independent Building Blocks (SIBs).

The CoreINAP Driver provides an implementation of the ETSI Core Intelligent Network Application Protocol (INAP) which is a Remote Operations Service Element (ROSE) user protocol. [7] The CoreINAP Driver connects to the underlying SS7 to communicate with other Service Points in the network. The Naming Service is a lightweight implementation of the CORBA Naming Service specification. The CoreXI provides a powerful generic request-and-response type interface to the database.

The CoreINAP Driver, Naming Service, and Database Driver components of the architecture are supplied by a third party vendor for the HP-UX 10.20 platform while the service specific factories, their associated call instance handling tasks as well as the SIB Inventory had been developed and execute on the Solaris 2.5 platform. Hence realizing the resulting framework lay in implementing the components which comprise the Service Logic Program (SLP) Factories, SLP Instances (SLPIs) and the SIB Inventory together with ensuring that they are designed to interact effectively via the IDL interfaces of the CoreINAP Driver, Naming Service, 
and Database Driver components. These will constitute the Service Logic Execution Environment as well as a significant proportion of the Service Creation Environment (SCE) [8].

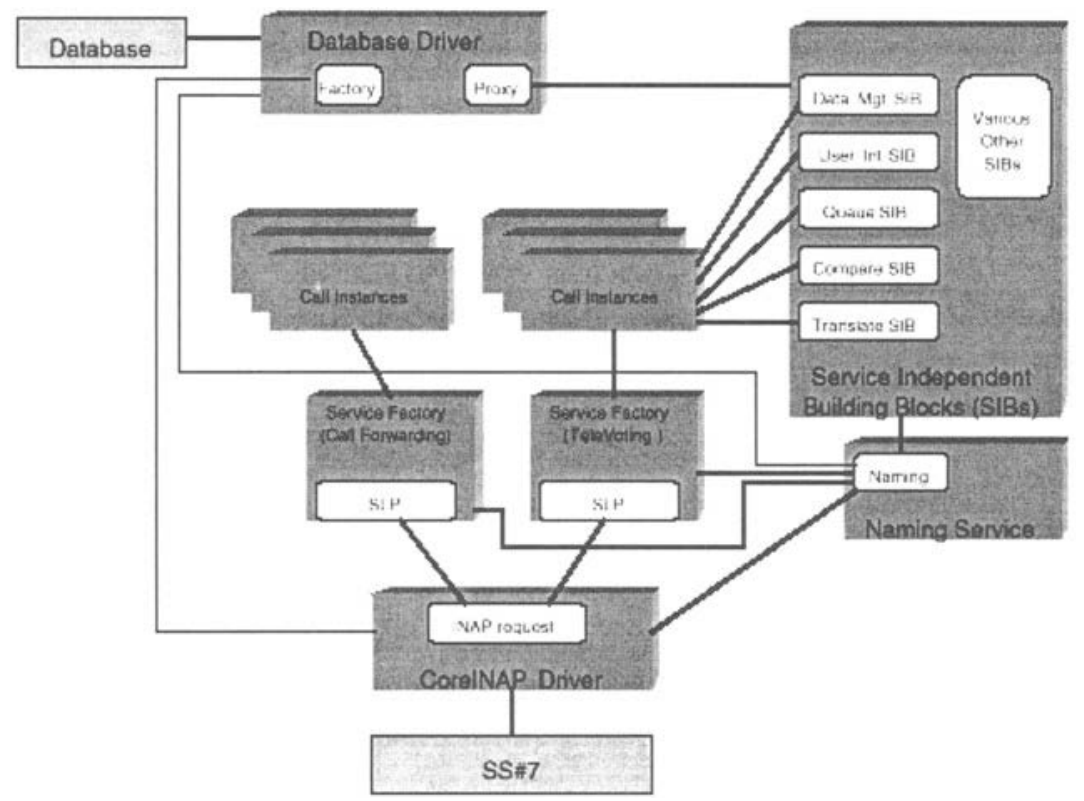

Figure 1. Framework Architecture

\subsection{The SLP Factory}

The design of the SLP Factory was aimed at supplying a service creator a generic class to derive his concrete service factories from. It furnishes an abstract interface for creating and destroying the various kinds of service logic instances. This provides consistency in the creation of new services and service factories. The interface also declares an abstract functionality of registration with the Naming Service which the instantiated service specific concrete factory can then override.

Apart from the above, it possesses a concrete interface together with operations for converting protocol-specific primitives (in this case INAP Operation primitives) into more generic information flows which can sufficiently supply the SLPIs as well as the SIBs with the information they need. Should the choice of the underlying protocol change, the propagation of the change would be greatly localized. The service designer needs only change the mapping element within the factory instead of having to redesign 
all services from scratch, as shown in Figure 2. Table 1 shows how the mapping is done between the two.

Table 1. Mapping of INAP Operations to Information Flows

\begin{tabular}{ll}
\hline INAP Operation & Information Flow \\
\hline Connect & Conn \\
ConnectToResource & ConnToRes \\
DisconnectForwardConnection & DisconnectForwardConnection \\
EstablishTemporaryConnection & EstablishTemporaryConnection \\
EventReportBCSM(oAbandon) & EndOfCall \\
EventReportBCSM(oAnswer) & CallAccept \\
EventReportBCSM(oCalledPartyBusy) & Busy \\
EventReportBCSM(oDisconnect) & EndOfCall \\
EventReportBCSM(oNoAnswer) & NoAnswer \\
EventReportBCSM(routeSelectFailure) & RouteSelectFailure \\
InitialDP & CallOrig \\
InitiateCallAttempt & InitiateCallAttempt \\
PlayAnnouncement & PlayAnnouncement \\
ReleaseCall & ReleaseCall \\
RequestReportBCSMEvent & NotifyBCSMEvents \\
\hline
\end{tabular}

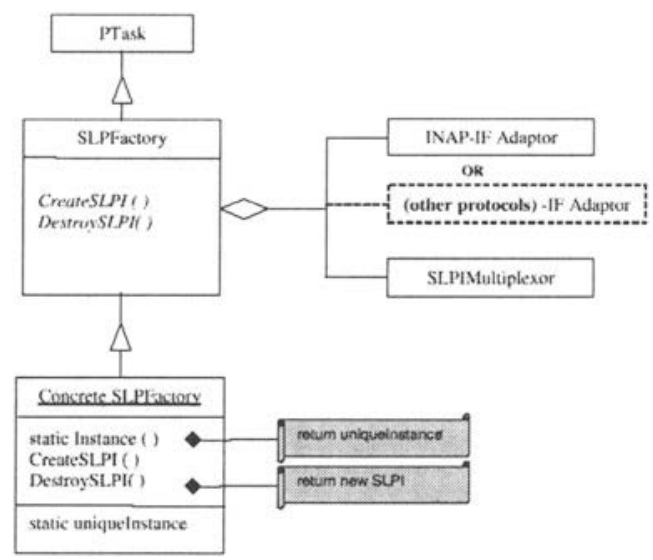

Figure 2. Creating a concrete SLP Factory

Lastly, the SLP Factory provides a concrete implementation of a multiplexing service to effectively handle the multiple instances of the services responding to the calls, one SLPI per call.

The implementation of the SLP Factory was achieved through a modified Abstract Factory Pattern [9] to take into account the INAP-specific and multiplexing nature of the resultant factory. Figure 2 illustrates the creation of a simple concrete Factory. The resulting concrete factories are usually 
enforced with the Singleton pattern, since every application typically needs only one instance of a concrete Service Factory per service.

It can be easily seen that many of the INAP Operations have a natural one-to-one correspondence to their respective mapped Information Flow elements. However, some composite INAP Operations such as EventReportBCSM are decomposed into their individual contextual and semantic representations at the Information Flow level. The usage of an Information Flow ideology stems from the potentiality to map the entire dataflow at the same level in the IN Conceptual Model (INCM) as the Distributed Functional Plane (DFP). Information flows in the DFP are between two functional entities which either consist of a client request/server response pair or of a client request alone [10].

The SLP Factory was also designed to present a unified viewpoint of the SCE and the only gateway to the protocol engineer for each particular service; all calls which require a particular service would be channelled to the single concrete SLPFactory for that service, which will subsequently reroute the call to a dedicated SLPI to handle that call.

\subsection{The SLP Instances}

The SLPTask class serves as a base from which all SLPIs are derived from. The role of the SLPTask is three-fold; it firstly provides the necessary support at the framework level needed by each call instance for proper service execution. It also acts as the Global Service Logic (GSL) which serves as a storage area for data, or glue, essential for the proper interaction and subsequent service realization by the SIBs, and lastly it partially models the BCP to provide Points of Initiation (POIs) and Points of Return (PORs).

SLPTasks are created and destroyed by the SLPFactory to which they belong to. Call instance routing is done from the SLPFactory to the SLPTask via the SLPTask's getID () function call, which returns a unique identifier with which the SLPFactory distinguishes each call instance.

\subsection{The SIB Inventory}

The SIB Inventory provides a subset of fully implemented SIBs which can be easily used as building blocks for a service. All SIBs within the inventory are implemented as objects which register themselves with the Naming Service upon startup. The SIB Inventory presently supports only synchronous communication techniques. The start() function call is responsible for launching the logic of the SIB. The test framework has implemented five of the fourteen SIBs defined: The Compare, Translate, 
Service Data Management SIB, the User Interface SIB and the Basic Call Process (BCP) [11].

One of the driving design issues of building a SIB Inventory is that the instantiation of the SIBs should be as minimal as possible to avoid a proliferation of an extremely large number of SIB instances for a moderate to complex service design. At any one point in time, service implementations, which would utilize several different kinds of SIBs, should ideally use only one of each kind of SIB. Thus the SIBs have been designed following the principles of design similar to the Flyweight Pattern [9].

However, the usage of CORBA means that the Flyweight Factory is no longer necessary, as it can be replaced by the ORB handling all operation invocations on the server SIB objects without any undue interference and with little loss in performance. All objects within the same server process on a given machine can be managed by the same process on that machine. Some ORBs such as Orbix also support an activation mode in which only one instance of a server object is launched to handle multiple client requests. In addition, if a server is registered in the shared mode, it can be launched manually, prior to any invocations on its objects [12].

The principles of how this model interacts and interworks with an SLPI (meaning an SLP instance of a concrete SLPTask-derived class) is described in the next subsection.

\subsection{Interworking between the SLP Instances and the SIB Inventory}

Since each SLPI is an event-driven task, it possesses state information and depending on the current state and the type of Information Flow (IF) it receives from its concrete service factory, it launches a predetermined chain of SIBs to perform the necessary series of actions. The SLPI thus forms the "glue" which holds all the necessary data and parameters needed by the SIBs, since the SIBs themselves are independent blocks which have no notion of service. Therefore at the GFP, the SLPI would also perform the functions of the GSL. At the Service Plane (SP) level, the entire service should appear seamlessly integrated, so clear IDL interfaces are needed for both the SIB implementations and the SLPI which need to be known at compile time for data to be interchanged efficiently to realize such IN services.

In order to interwork harmoniously, following the flyweight pattern, the SLPIs would behave as clients while each SIB would be represented as a ConcreteFlyweight, each having a similar interface to the others, differing only in terms of the names used to register with the Naming Service. However, flyweights cannot assume the context in which they are operating. 
As the SIBs will be unable to store any extrinsic information, all data uniquely pertaining to the entire duration of a call, or to a temporally specific moment within the call, need to be held by the client, which in this case refers to the SLPI. Such data would usually involve the CID and SSD parameters for use by all the SIBs. The next subsection will describe how the SSD is stored within the SLPI.

\subsubsection{Storage of the SSD in the SLPI}

The variable information which the SLPI needs to store varies not only with the state of the SLPI, but also with the type of input the SLPI receives. Thus if an SLP Instance has two states, Idle and Active, and is able to receive two kinds of Information Flows, Call_Begin and Call_End, a total of four different SIB chain combinations need to be defined.

The State Pattern [9] when modified, provides an easy behavioural technique for managing this complexity. The key idea is to introduce an abstract class called SIBChain which represents the state*input combinations of the SLP Instance. The SIBChain class declares an interface common to all the classes that represent the different operational permutations. Subclasses of the SIBChain implement the specific combinatorial behaviour. For example, the classes Idle_Call_Begin and Active_Call_Begin specify and implement the behaviour pertinent to that particular SIB chain. Figure 3 illustrates how this was accomplished.

The classes can also be shared between SLPIs using the Singleton Pattern. However, the introduction of the SIBChain by itself does not solve the problem of SSD storage. Rather, it provides a logical breakdown of the different numbers of SIB chains necessary for the service execution. Also, by virtue of its semantics, the SSD will change each time the SIB is reused or changed, and hence, a more dynamic form of representation is necessary for such a storage. This therefore leads to the usage of proxy objects for the SIB flyweights, the SIBNodes.

By using node pointers, inheritance as well as SIB-specific internal data representation to store the SSD of the various SIBs they represent, the SIBNodes present a common interface to the SLPI to access the SSD needed by the next SIB in the chain when it is requested, and systematically present an ordered and organized way to specify the service in terms of its SSD. Five of these proxy SIBNodes are implemented, corresponding to the five SIBs implemented within the SIB Inventory. The following illustrations show the object diagram depicting this. 


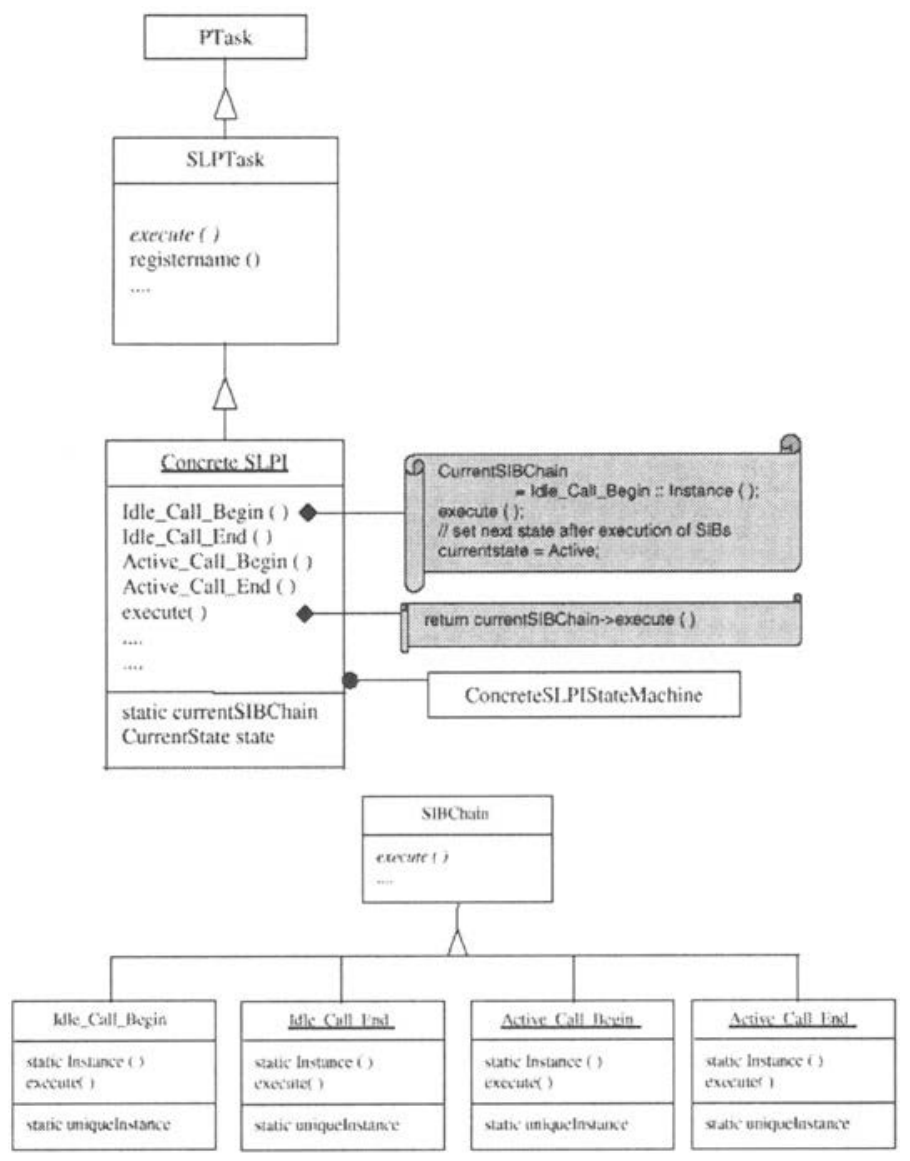

Figure 3. Usage of the modified State Pattern

Each SIBChain derived class is responsible for the construction of a treelike branching chain using these SIBNode derived classes upon creation, and then initializing them to contain the relevant SSD information used for the proper execution by the SIBs upon reaching that level. However, it remains the SLPTask's responsibility for maintaining the current status of processing within that tree. Upon completion of processing, the current SIB will request the SLPTask for information of the next SIB to which control should be passed through the SLPTask's getnextsib function call. This call will return the next SIB and its SSD in the chain, depending of course on the results of execution by the current SIB. Thus, using such a technique, a service designer is able to organize session specific data in a manner logical and organized enough for the SLPTask to control, manage and interpret while being able to interwork with the necessary SIBs. 


\subsubsection{Storage of the CID in the SLPI}

The storage and retrieval of CID per SLPI by the SIBs proceed in a different manner as compared to the complexity of managing the SSD. Far from being context-specific, the information stored as CID does not change for the duration of the call instance.

Thus the best technique possible to store the CID would be within the SLPI itself, with the SLPTask providing an interface with which the SIB can retrieve or manipulate the CID using direct object calls. Currently the framework implementation supports, implements and returns such pertinent function calls made by the SIBs in the Inventory to the SLPTask requesting call instance specific data such as the called party number as well as the calling party number. Other kinds of CID operations are left as virtual operations for implementation at the service designer's liberty.

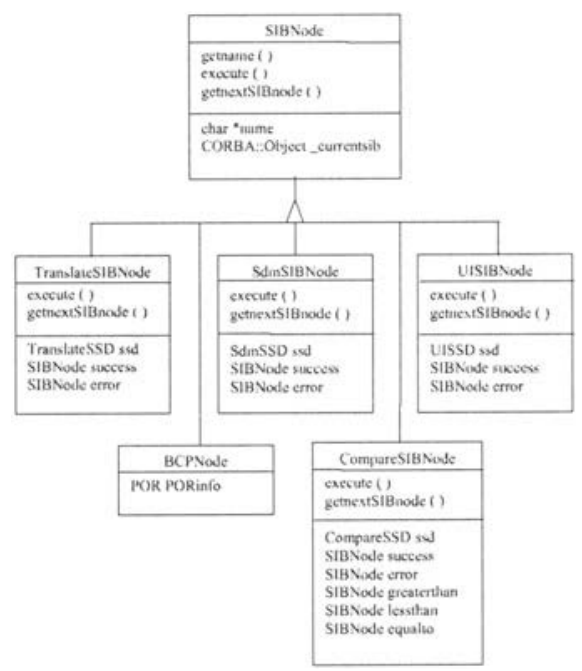

Figure 4. SIBNode class derivation

\section{AN EXAMPLE SERVICE CREATION SCENARIO}

The Number Translation Service (NTS) is a simple service where numbers beginning with a certain sequence trigger off IN service processing logic. The service basically centers around 4 different phone numbers: numbers $\mathrm{A}, \mathrm{B}, \mathrm{C}$ and $\mathrm{D}$. As an example, if someone telephones number $\mathrm{A}$, the call will be rerouted to either $\mathrm{B}, \mathrm{C}$ or $\mathrm{D}$, depending whether it is during working hours, after working hours or during a holiday. If the rerouted number is unreachable, busy or unanswered, the service logic would then 
proceed to connect the caller to a default number. As such, the NTS service logic needs to make queries to the database to obtain the correct number to connect to in the telephone network.

The functionality of the SIB chain that is to be launched upon reception of the CallOrig IF in the Closed state is shown in Figure 5. Three SIBs from the SIB Inventory were used to realize this particular SIB Chain, with the CompareSIB and the TranslateSIB being used multiple times.

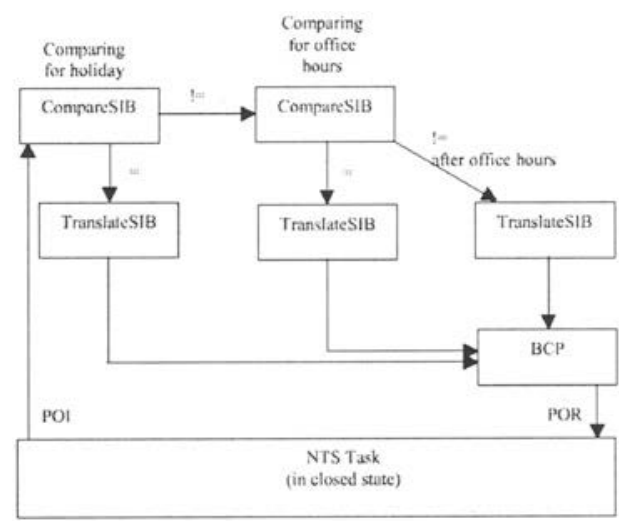

Figure 5. Chained SIBs upon receiving the CallOrig IF

\section{DESIGN DECISIONS}

The framework presents a model in which complexity is hidden via base classes and a single unified interface is presented at key access points to its components. As a consequence, the entire SLEE is viewed at from only one viewpoint from the underlying application specific protocols such as INAP. Such principles of component oriented programming have proven extremely useful in managing and reducing the problem domain into clear and exclusive subdomains which could then be tackled easily.

Most, if not all, of the Design Patterns used in this work would have been of little use if they had been used "as is". In some cases, modifications to harness the distributed nature of the environment were highly necessary. However, once altered and adapted to work with such distribution, they seemed to support and work seamlessly with the rest of the design of the system.

The modified State Pattern distributes behaviour for the different combinations across several subclasses. This increases the number of classes and is less compact than a single class. However, such a distribution is 
actually good, because given a large permutation, the only other way to solve it in a single class would be to necessitate large conditional statements. The usage of the State Pattern was preferred over the more traditional tablebased alternative due to the fact that this pattern allows the localization of state-specific behaviour into one object. An added bonus is that since state objects can be shared if they have no instance variables, they essentially become flyweights with no instrinsic behaviour.

Often, such State Patterns are combined with the Singleton. However, the drawback here of employing the Singleton Pattern with the State Pattern is the one basic premise that class instances created by enforcing the design technique introduced in that particular pattern are expected to be persistent throughout the application. This might not be appropriate for the classes adhering to the modified State Pattern, as they need only exist as long as concrete SLPIs implementing the service and representing Call Instances exist. Otherwise they should be deleted. Many algorithms can be proposed to implement such a scheme. Perhaps the most straightforward would be a modified Singleton with a relatively simple reference-counting garbage collection technique. This technique was employed successfully in building the example Number Translation Service (NTS).

The concepts introduced by the utilization of the Information Flows and the BCP were applied liberally. It allows the framework to be robust enough to withstand variations in underlying protocol services as well as being capable of extending towards rudimentary "Plug-and-Play" type support at the protocol level. Information Flows furnish a more natural way of modeling service logic input than INAP service primitives provide.

Modeling the BCP could not be easily achieved by treating the SLPTask as a BCP by itself. Thus the BCP was modeled in a fashion similar to other SIBs, the difference being that the BCP defines the PORs by which the SIB chain ends. The PORs were mapped into function calls in the SLPTask, thereby bringing control gracefully back from the SIB chain. From this perspective, POIs could be easily mapped into events, but PORs were much better mapped as function calls. Keeping the SIBs purely synchronous resulted in a set of objects whose co-operation was easier to manage.

The design of the SIB and abstract SLP classes are done in such a way that interworking between the SIB Inventory and the concrete SLPIs can be achieved without much difficulty. However, close co-operation is needed at least at the framework level to be able to successfully achieve mapping on both the GFP and the SP in the INCM. Although in principle many services other than the NTS can be implemented with these SIBs such as those described in [13], the application programming interface for the SIBs was not specified in IN CS-1. One other issue which also lay in mapping the different planes of the INCM was the fact that the entire specified model has 
been mostly based on a functional and modular data flow approach. This made the task of denoting the same relationship with objects that express such associations as clearly, open to some interpretation.

However, although SIBs were specified as abstract entities which must be decomposed into a set of interacting FEs and FEAs at the DFP, the experience gained from this work shows that using an object oriented model, the realization of SIBs as distinct and concrete implementations which provide unified and stable interfaces is possible as the usage of CORBA technology has led to a credible usage of these building blocks.

Subsequently, the prototyping of simple IN services can be achieved extremely rapidly as compared to more traditional SCEs at which reuse may lie either at the network or application resource level. It may not be unrealistic to note that the development time schedule for the implementation of fully working IN services may be reduced orders of magnitudes using a framework built with a similar design objective as this.

\section{CONCLUSIONS}

The presented framework is compliant with the core CORBA 2.2 specifications [14] as well as the INCM. Conformance to the TINA model can be observed within the confined context of CS-1. A one-to-one mapping between the INCM and the TINA conceptual framework is not entirely possible, but several parallels can be drawn from both models which allow a partial mapping, such as the mapping of the SIBs to TINA service objects/logic, as well as the mapping of some TINA computational objects at both the DFP and the GFP [15]. In the long run, it must be borne in mind that the IN Capability Sets are still evolving.

The OMG working group for IN/CORBA Interworking has proposed two orthogonal scenarios of using CORBA in IN Signaling. The first may be identified as a gateway which allows CORBA-based IN application entities to interwork with those that communicate using the INAP, while the second would involve using the existing Signalling System No.7 (SS7) infrastructure as a transport network for GIOP messages[16]. Of the two, the first type of interworking, which is what this framework has attempted, is expected to be the more prevalent of the two as a principal and immediate solution.

In conclusion, it is imperative to mention that interworking between CORBA and Intelligent Networks Systems is still under active study and the work presented in this paper uses, discusses and attempts to find a working solution based on these concepts and ideas. 


\section{REFERENCES}

[1] Intellitel Communications: White Paper on Network Intelligence Middleware, 1999

[2] D. C. Schmidt: Reactor: An Object Behavioural Pattern for Concurrent Event Demultiplexing and Event Handler Dispatching. Pattern Languages of Program Design (J.O. Coplien and D. C. Schmidt, eds.), Reading, MA: Addison-Wesley, 1995.

[3] B. Silverajan, J. Harju: OVOPS - Enhancing an Event Based OO Framework for Distributed Programming, Proc. TOOLS USA '99, Santa Barbara, CA August 1 - 5, 1999.

[4] OMG : CORBAservices, Common Object Services Specification, December 1998

[5] OMG : Messaging Service Document orbos/98-03-12

[6] OMG : Notification Service Document telecom/98-01-01

[7] ETSI: Recommendations ETS 300 374-1, Intelligent Network Capability Set 1 (CS1) Core Intelligent Network Application Protocol (INAP) Part 1: Protocol Specification, ETSI 1994.

[8] B. Silverajan: A CORBA-Enhanced Implementation Environment for Distributed IN Service Creation, MSc Thesis, 1998.

[9] E. Gamma, R. Helm, R. Johnson, J. Vlissides: Design Patterns, Elements of Reusable Object-Oriented Software. Addison-Wesley 1995.

[10] ITU-T Recommendation Q.1204 : Intelligent Network Distributed Functional Plane Architecture, 1993

[11] ITU-T Recommendation Q.1213 : Global Functional Plane For Intelligent Network CS-1, 1993

[12] IONA Technologies : Orbix 2.2 Reference Guide, March 1997

[13] ITU-T Recommendation Q.1211 : Introduction to Intelligent Network Capability Set 1, 1993

[14] OMG: The Common Object Request Broker: Architecture and Specification. CORBA V2.2, February 1998

[15] Demounem, L.: Intelligent Networks and TINA Service Models - Present and Future, Proceedings of the IFIP/ICCC International Conference on Information Network and Data Communication (INDC '96), Trondheim, 1996

[16] OMG: IN/CORBA Interworking, document telecom/98-10-03 